\title{
The Current Pratices, Training and Concerns of a Group of Hospital-Based Speech Therapists Working in the Area of Dysphagia
}

\author{
Naina Modi and Eleanor Ross
}

Department of Speech Pathology and Audiology

University of the Witwatersrand

\begin{abstract}
The aim of the study was to investigate the current practices, training, and concerns of a group of hospital-based speech therapists regarding the area of dysphagia practice. Questionnaires were posted to 75 speech therapists working in the major hospitals around Gauteng, Kwa-Zulu Natal and the Cape Province and responses were obtained from 50 therapists within the stipulated time. Closed-ended questions were analysed quantitatively using descriptive statistics, while openended items were subjected to content analysis of themes articulated by respondents. Results indicated that the overwhelming majority of the respondents were involved in assessing and treating dysphagia patients. Specific problems faced by respondents included limited staff to share their workloads, large caseloads, restricted access to supervision / mentor systems and poor knowledge by other health personnel of their role in dysphagia. Almost half of the respondents also reported that they were involved in student training which included the area of dysphagia. Investigation of respondents' undergraduate and postgraduate training in dysphagia suggested that there were gaps in the theoretical content of these courses as well as a limited focus on practical training. Another finding was that respondents showed an awareness of the specific roles of the speech therapist in dysphagia intervention as well as the roles of the professionals constituting a multidisciplinary dysphagia team. Most of the respondents reported that they had access to dysphagia team members as well as to diet modification services. However, many of the respondents showed decreased knowledge of and awareness regarding accessibility of diagnostic procedures and suctioning methods used in dysphagia. The majority of the respondents rated their knowledge about specific theoretical areas as well as their counselling abilities to be better than their actual skills and confidence in assessing and treating dysphagia patients. Implications of the study included the need for clinicians, universities and professional organizations to work together to improve the existing situation.
\end{abstract}

KEY WORDS: dysphagia; practices; training; hospital-based speech therapists

\section{INTRODUCTION}

Of all the areas of work of a speech-language pathologist (SLP), dysphagia constitutes the most prominent single area where improper management places patients at risk for physical impairment or even death (Cannito, 1995). Dysphagia is a disorder of swallowing and refers to the difficulty in moving food efficiently through the oral, pharyngeal or oesophageal areas. Dysphagia symptoms may include aspiration, slowed or laboured eating, weight loss, coughing during eating, frequent throat clearing or expectoration, a sensation of swallowed material being retained in the throat, and pneumonia. The patient may also exhibit an inability to keep food in the oesophagus or stomach resulting in reflux (Logemann, 1998). While no figures are currently available for the South African population, it is estimated that 6-10 million Americans suffer from some degree of dysphagia.

Speech Language Pathologists (SLPs) have been evaluating and managing patients with oropharyngeal dysphagia since the 1930 s when clinicians began to work with patients whose oral motor control for speech and swallowing was compromised as a result of cerebral palsy. Since then, the involvement of SLPs in dysphagia has grown steadily, particularly in the United States (Logemann, 1998). The 1993 ASHA Omnibus Survey (cited in Martin, 1995) revealed that approximately $60 \%$ of practising SLPs in health care settings throughout the United States were involved in dysphagia intervention. The role of the SLP has also expanded beyond providing assessment and treatment to the overall management of the patient's nutrition and pulmonary health, thereby dealing with severe and complicated problems (Langmore, 1991).

From the possible complications that can occur as a result of dysphagia, aspiration is most significant clinically. When the airway is unprotected and foreign material is aspirated into the lungs, the patient is at risk of developing pulmonary infection and aspiration pneumonia, a disease that has been identified as the fifth leading overall cause of mortality in the United States (Cannito, 1995).

The life-threatening nature of dysphagia intervention thus highlights the need for a high standard of service provision. In recognition of this need, ASHA has revised many of its policy documents defining the scope of practice; 
the preferred practice patterns; position statements; and practice guidelines specifically related to dysphagia (ASHA, 1993). Detailed proficiencies, knowledge and skills have been outlined not only by ASHA (1989), but also by the College of Speech Therapists (CST, 1990) as well as the Australian Association of Speech and Hearing (AASH, 1994).

These competencies relate to ensuring effectiveness in the various aspects of dysphagia intervention including: identifying individuals at risk for dysphagia; conducting clinical oropharyngeal and respiratory examinations with detailed case history information; undertaking instrumental structural physiologic examinations with related professionals; determining management decisions regarding methods of oral intake, risk precautions and treatment strategies with other professionals; providing education, counselling and training for patients, their families and other members of the dysphagia team; participating in an interdisciplinary dysphagia team - which requires knowledge of the roles of different team members and facilitating team communication, and teaching and supervising supportive personnel and students in training - which requires the ability to identify education and clinical training needs and to provide effective supervision and training.

Essential resources for quality care have also been identified by ASHA (1989), CST (1990) and AASH (1994). These include the availability of allied health professionals who play a critical role in establishing and sustaining a successful dysphagia programme; adequate communication channels amongst the health professionals in the dysphagia team; access to basic appropriate equipment such as gloves, spatulas and torches; availability of suction equipment during oral intake trials; access to specific programmes for non-oral feeding intervention, and facilities for diet modification (AASH, 1994).

This delineation of the competencies required for high quality dysphagia treatment has served to highlight the main obstacles faced by practising SLPs. There has been a general finding in the United States, United Kingdom and Australia that most undergraduate speech pathology courses have not adequately equipped graduates with sufficient knowledge and skills in the area of dysphagia (AASH, 1994; Martin, 1995). Dysphagia has typically been integrated into existing courses resulting in an overwhelming body of information and a decreased amount of hands-on experience and observations (Cannito, 1995). SLPS have been found to be lacking in knowledge of concepts relating to human physiology and seem to have only a vague appreciation of surgical techniques, laboratory data, radiological protocols, medical procedures as well as the roles of various members of the dysphagia team (Miller, 1995). Much difficulty has been experienced in working with patients in critical care units where continuous cardiopulmonary monitoring occurs. Lack of training in this area thus leads to feelings of intimidation and incompetence in treating the critical care patient (Martin, 1995).

The amount of closely supervised hands-on experience under qualified professionals with demonstrated expertise in dysphagia has also been found to be lacking (Miller, 1995) due to the shortage of, or under-utilisation of suitable medically-based practicum sites (Groher, 1995). With the high demand for services for both paediatric and adult dysphagia in medical settings, clinicians have been forced to improve their knowledge and skills through alternate sources such as conferences and workshops run by colleagues with self-acquired clinical expertise, through journals and books, and through observing dysphagia clinicians in medical settings (Martin, 1995). However, the availability of such resources is limited and most conferences and workshops tend to be short 1-2 day courses which offer limited opportunities to develop practical skills (CST, 1990). These limitations lend themselves to questioning the efficacy of SLPs' intervention with dysphagia. Logemann (1998) suggests that the ultimate test of efficacy relates to the number of patients who are managed effectively and the percentage of hospitals that exhibit multidisciplinary dysphagia programmes.

Several steps have been taken to identify possible solutions for the gaps in dysphagia training and intervention. Martin (1995) emphasises the important role of position statements and guidelines which clearly outline the proficiencies, knowledge bases and competencies required for the effective provision of services in specific areas. She also describes the benefits of creating special interest groups where SLPs with similar clinical and research interests are able to interact and exchange information on a regular, structured basis. Specific plans should also be developed in order to recognise individuals demonstrating a particular expertise in an area of clinical practice. This goal could, for example, be achieved by developing a dysphagia speciality certification programme which recognises such professionals and utilises their services as clinical competency instructors for novice clinicians in the field (Martin, 1995). The development of a comprehensive graduate core curriculum for speech pathology programmes in universities around the country is likely to ensure that all SLPs are able to demonstrate basic clinical competencies in dysphagia management before actually treating medical patients (Martin, 1995).

It is evident from the literature that ASHA, AASH as well as the CST are all actively involved in trying to implement the above recommendations. A position paper of the CST drafted in 1990 outlines the recommended levels of training required for undergraduate and postgraduate studies in dysphagia. Detailed information is provided on specific objectives, aims, course content and clinical experience to be achieved at the undergraduate and postgraduate levels. Similarly, the AASH position paper (1994) clearly describes the knowledge and skills needed for effective assessment and treatment as well as for exercising necessary safety precautions related to cross-infection and emergency situations. The Ad Hoc Committee on Dysphagia (ASHA, 1987) outlined the guidelines for adequate clinical preparation needed to conduct competent evaluation and management of dysphagia. Specific attention was also paid to the SLP's scope of practice in relation to instrumental diagnostic procedures for swallowing, including videofluroscopy, pharyngeal manometry, manoflurography, ultrasonography, scintigraphy and electromyography (ASHA, 1991).

In summary, countries such as the United States, United Kingdom and Australia, have identified the following key components that comprise an acceptable standard of service in dysphagia:

a) Adequate training in dysphagia at an undergraduate and postgraduate level including: (i) a separate detailed module on dysphagia; (ii) supervised hands-on practical training at medically-based practicum sites; and (iii) 
experience with working on an interdisciplinary level

b) Adequate knowledge of $S L P$ s regarding: (i) human anatomy related to dysphagia; (ii) risk factors in dysphagia; (iii) instrumental procedures used in dysphagia assessment and intervention; (iv) the roles of the key members in a dysphagia team; and ( $v$ ) medical terminology and conditions associated with dysphagia

c) Access to essential resources in the work setting including:

(i) appropiriate equipment; (ii) diet modification services; (iii) and key members of the dysphagia team

In view of the nature of the problems identified in these developed countries, it was postulated that similar problems were probably being experienced by SLPs working in South Africa. Training for a career in Speech Pathology is limited to selective larger universities throughout South Africa and the typical size of such departments is relatively small in contrast to other medical and paramedical professions. A separate course in dysphagia may thus not be common in these departments due to financial constraints. Furthermore, not all of these departments may be comprehensively linked to a medically-based practicum site. The knowledge and experience gained by SLPs may thus be inadequate in a number of areas including instrumental evaluations, teamwork, medical conditions as well as oropharyngeal anatomy. In spite of the fact that therapists may feel inexperienced and incompetent in dealing with dysphagia patients, they may be unable to retract their services due to a lack of manpower and also due to the fact that they still appear to know more about dysphagia than many other health professionals. Furthermore, in view of the general economic crisis currently confronting the South African health sector, SLPs are also being faced with limited resources in terms of support services and equipment (White Paper for the Transformation of the Health System in South Africa, 1997).

While the role of the SLP in the treatment of dysphagia is recognised in many countries, it was envisaged that the same might not be occurring in the South African situation. According to the Preliminary Draft of the Policy Document for Communication Professionals (1993) in South Africa, individuals with dysphagia were not included as a separate client group, suggesting that at the time in South Africa, the role of the SLP in dysphagia treatment was not well recognised both within and outside the profession.

The neglect of dysphagia may also be partly related to current health care policy as articulated in the White Paper for the Transformation of the Health System in South Africa (1997), which emphasises a primary health care approach. Dysphagia treatment, on the other hand, utilises expensive high technology equipment and requires high levels of expertise, thus falling within the ambit of tertiary health care. Nevertheless, the risks for pulmonary infections and aspiration pneumonia coupled with high mortality rates, make dysphagia intervention of critical importance.

Educational standards, access to services and standards of facilities all contribute to the process of quality assurance, yet research into dysphagia training and practice among SLPs in the South African context has been extremely limited with few studies focusing specifically on dysphagia training and practice. Information about the extent of involvement of South African SLPs in dysphagia intervention is important, but even more crucial than this aspect is the standard and quality of current dysphagia practices.
By investigating clinicians' knowledge and concerns regarding dysphagia intervention and linking this information to their training and practical experience, it was anticipated that major gaps might be identified in present undergraduate and postgraduate training curricula. It was further envisaged that insight into the types of obstacles faced by practising clinicians might highlight factors preventing comprehensive dysphagia intervention. This type of information is vital for therapists motivating for equipment and staff.

In view of the fact that the South African SpeechLanguage Hearing Association (SASLHA) was involved with drawing up a position paper on dysphagia at the time the present study was undertaken (SASLHA, 1996), it was considered useful to gain insight into the training, current practices, knowledge and fears of SLPs working in dysphagia. The present study thus aimed to investigate these areas amongst hospital-based SLPs in South Africa so that the information could be fed back to the professional body and relevant academic institutions.

\section{METHODOLOGY}

\section{$A I M$}

\section{Main Aim}

The overall purpose of the research project was to explore the current practices, training, knowledge and concerns of a sample of SLPs working in hospitals regarding dysphagia intervention.

\section{Sub-Aims:}

1. To explore current practices of a group of hospital-based SLPs with regard to dysphagia management.

2. To investigate the undergraduate and postgraduate training of respondents with respect to dysphagia.

3 . To elicit respondents' views regarding the role of the speech therapist in the dysphagia management team.

4. To investigate respondents' knowledge and perceptions of multidisciplinary teamwork in dysphagia management.

5. To obtain information on respondents' access to essential resources

6. To explore their self-perceived levels of professional competence and emotional comfort with regard to the area of dysphagia.

7. To explore respondents' general views and concerns regarding the management of dysphagia.

\section{RESEARCH DESIGN}

The research project was undertaken in the form of a cross-sectional survey employing a postal questionnaire.

\section{SUBJECTS}

A convenience sample was surveyed which comprised 75 speech-language pathologists (SLPs) working at 24 provincial hospitals in Gauteng, Kwa-Zulu Natal and the Cape Province. It was assumed that these therapists would probably have more contact with dysphagia patients than therapists working in other settings, and that the complexities involved in managing dysphagia patients might be more 
obvious in an acute care setting where life-threatening medical problems often co-exist with dysphagia.

\section{PROCEDURE}

\section{Research instrumentation}

In view of the cost implications related to the large sample size and wide geographical distribution of the respondents, a postal survey was chosen as the most appropriate research method for the purposes of this study. A copy of the questionnaire is available from the authors on request. The questionnaire comprised seven sections:

Section A: Biographical data. The aim of this section of the questionnaire was to obtain data on factors such as year and university of graduation as well as amount of work experience in a hospital setting.

Section B : Current practices in dysphagia management - The aim of this section was to obtain information about the number of speech therapists working in respondents' departments; respondents' involvement in the area of 'dysphagia management; respondents' involvement in student training; involvement of students in dysphagia intervention, and sources of referral of dysphagia patients.

Section C: Training in dysphagia. This section was divided into two sub-sections. Questions in the first section related to respondents' undergraduate training in dysphagia intervention. Perceptions of the adequacy of their training were assessed via a five-point rating scale. The second part of this section focused on respondents' postgraduate training.

Section D: Speech therapists' role in dysphagia management . The first part of this section aimed to investigate respondents' perceptions of the specific roles of the speech therapist in dysphagia intervention. An additional aim of this section was to investigate respondents' perceptions about whether this role was recognised by their medical and paramedical colleagues. The second part focused on respondents' perceptions and knowledge about teamwork as it relates to dysphagia intervention as multidisciplinary teamwork is strongly advocated in the literature as being the most appropriate model of care in dysphagia (Logemann, 1990).

Section E: Access to resources - Access to resources has been identified as one of the key elements of an effective service. The questions in this section thus aimed to obtain information about respondents' access to various diagnostic procedures, diet modification services, suction equipment, other health professionals and a system of supervision and mentoring.

Section F : Knowledge and competence - Therapists' selfperceived knowledge and competence in the area of dysphagia are likely to affect their comfort and confidence levels in handling dysphagia patients and counselling their families. Respondents' were thus asked to rate their knowledge, skills and feelings in relation to specific areas of dysphagia on a five-point rating scale.

Section G: General views on management of dysphagia This section consisted of further open-ended questions to elicit information regarding respondents' general concerns and comments about the area of dysphagia as well as their suggestions for overcoming these concerns.

\section{Pilot study}

The questionnaire was pre-tested on three SLPs who had previously worked in hospital settings, but were excluded

TABLE 1: Description of the subjects $(\mathbf{N}=50)$

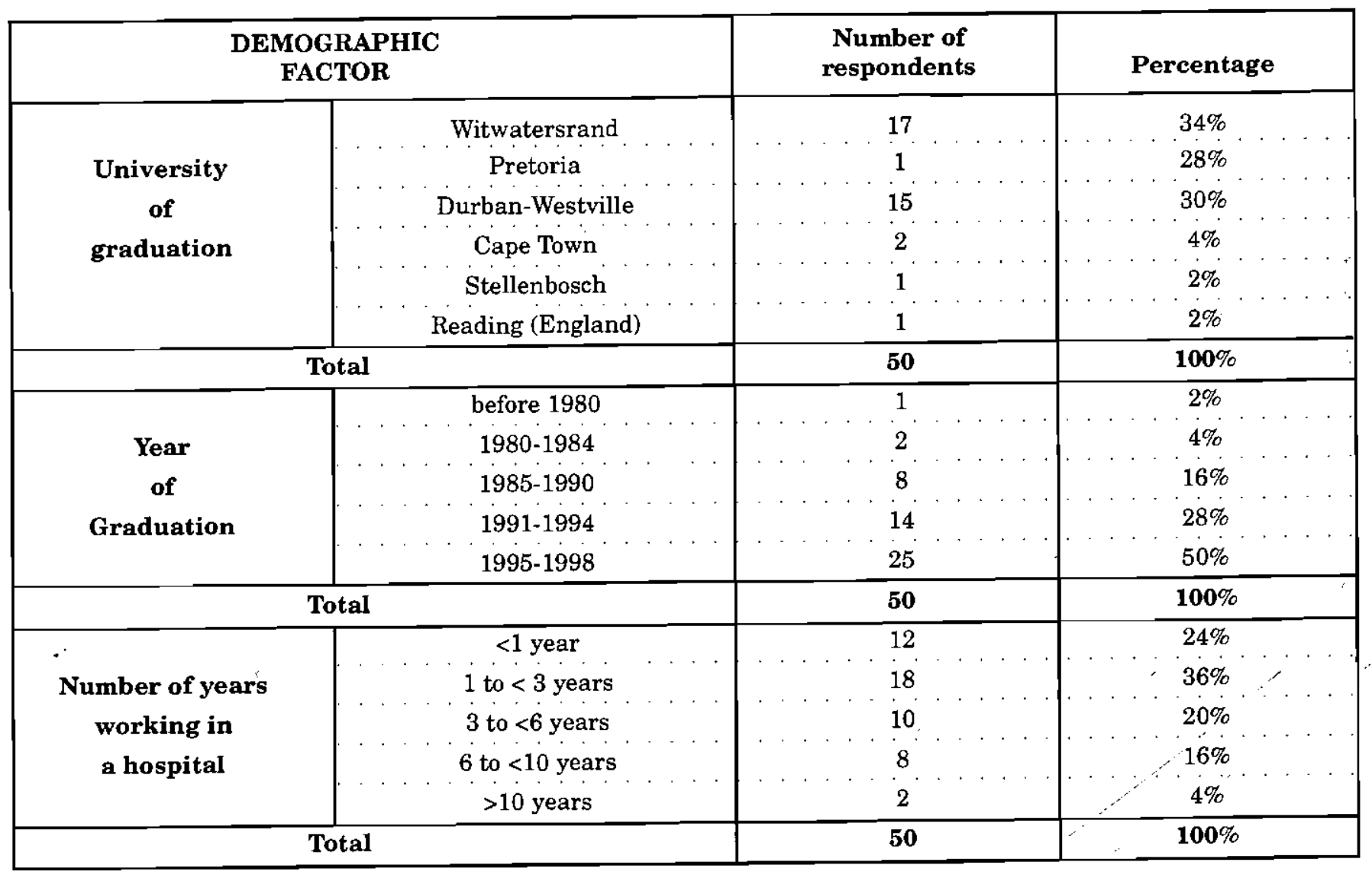


from participation in the field study. Based on the results of the pilot study, various changes were made to the questionnaire.

\section{Field study}

The heads of the Speech Therapy Departments at the 24 hospitals involved were thus contacted telephonically. Information about the purpose of the study was provided and the number of therapists working in each Department was confirmed. Questionnaires were then posted to the various Departments and due dates for their return was stipulated in the cover letters. A follow-up telephone call was then made approximately one week later to ascertain whether the departments had received the questionnaires.

\section{Data analysis}

Descriptive statistics were employed to analyse the responses obtained from the closed-ended items in the questionnaire, while content analysis was used to analyse open-ended questions qualitatively according to themes expressed by respondents.

\section{RESULTS AND DISCUSSION}

Self-administered questionnaires were sent out to 75 SLPs working in the major provincial hospitals around Gauteng, Kwa-Zulu Natal and the Cape Province, from which a $67 \%$ response rate was obtained. Results are discussed in accordance with the sub-aims of the study.

\section{CURRENT PRACTICES}

Investigation of respondents' current practices in dysphagia revealed that the overwhelming majority of respondents $(90 \%)$ were involved in assessing and treating dysphagia patients. A minimum of 1-5 patients were seen by most respondents $(62 \%)$ on a monthly basis. Almost half $(46 \%)$ of the respondents also reported that they were involved in student training and this training included the area of dysphagia management. These findings highlighted the need for respondents to be adequately trained in dysphagia practice at an undergraduate level.

This need was further emphasised by the fact that many respondents were working in small departments that had a limited number of more experienced senior staff. Opportunities for supervision and mentoring were thus thought to be limited. The results indicated that the majority of the respondents $(56 \%)$ were working in small departments consisting of only 1-4 therapists. When this fact was related to the years of working experience of these therapists, it emerged that almost one-fifth (18\%) of the respondents were working in departments where all the therapists had less than three years' working experience i.e. they were all junior therapists. Even more disconcerting was the finding that $8 \%$ of these respondents were working alone in their departments with no support from other junior or more senior experienced staff. These results suggested that many of the respondents might have been experiencing difficulty in obtaining the necessary support and guidance from more experienced or senior colleagues. It was therefore recommended that efforts be made to organise such supervision/mentor systems in conjunction with other hospitals and with university staff members. The need for supervision has been highlighted as an ethical responsibility for clinicians with limited experience (AASH, 1994: SASHLA, 1998)

A further finding in this section was that respondents received most referrals of dysphagia patients from doctors $(82 \%)$ and considerably fewer referrals from allied health disciplines. This finding suggested poor knowledge of the role of SLPs in dysphagia by other health professionals. The need for SLPs to advocate their role and services in dysphagia as well as to educate health professionals was thus highlighted.

\section{THE TRAINING OF RESPONDENTS IN DYSPHAGIA}

\section{Undergraduate Training}

Results displayed in Table 2 indicate that dysphagia was covered in the vast majority (96\%) of the respondents' undergraduate courses. Amost disconcerting finding related to two respondents who reported that they were actively involved in assessing and treating dysphagia patients despite not having received any basic training in dysphagia at an undergraduate level. Although both respondents did report attendance at continuing education courses and workshops, the exact level of this input could not be ascertained. This finding has serious implications as the Code of Ethics (SASLHA, 1997) clearly stipulates that SLPs should provide only those services for which they hold the appropriate qualification and for which they are competent by training. A lack of adherence to this principle is potentially harmful to the client, particularly in the area of dysphagia practice where improper management may have life-threatening consequences (Cannito, 1995).

Within the group of 48 respondents who received dysphagia training in their undergraduate studies, more than two-thirds (73\%) reported that dysphagia was not

TABLE 2: Dysphagia courses in undergraduate training

\begin{tabular}{|l|cc|}
\cline { 2 - 3 } \multicolumn{1}{c|}{} & \multicolumn{2}{c|}{$\begin{array}{c}\text { Dysphagia included in } \\
\text { Undergraduate courses }\end{array}$} \\
\hline $\mathrm{N}=50$ & NO & YES \\
\hline No. & 2 & 48 \\
\hline Percent & $4 \%$ & $96 \%$ \\
\hline
\end{tabular}

Whether dysphagia was included as a separate or integrated course

\begin{tabular}{|l|c|c|}
\hline N=48 & Number & Percent \\
\hline $\begin{array}{l}\text { Separate } \\
\text { module }\end{array}$ & 13 & $27 \%$ \\
\hline $\begin{array}{l}\text { Integrated } \\
\text { course }\end{array}$ & 35 & $73 \%$ \\
\hline
\end{tabular}


taught as a separate module, but rather integrated into other speech pathology courses. Toner (1995) suggests that this integration of the dysphagia module is typical in small speech therapy departments where the teaching load already exceeds university recommendations and therefore precludes the addition of new courses. He further states that this situation frequently results in a focus on theory with limited time for observation and hands-on experience, and therefore recommends that a separate course in dysphagia is a necessity.

Approximately half (48\%) of the respondents reported that their undergraduate speech therapy departments had not been linked to medically-based practicum sites. However, students are generally required to complete a clinical block at a hospital setting, thus it can be assumed that dysphagia practice is incorporated into the student training by such hospital-based clinicians.

With regard to the theoretical areas in the undergraduate courses as suggested by ASHA (1989), CST (1990) and AASH (1994), most of the respondents were apparently taught about the anatomy and physiology of swallowing (86\%), medical conditions and disorders related to swallowing (73\%), assessment methods in dysphagia (79\%) and management procedures in dysphagia ( $75 \%$ ). However, the areas of legal and ethical issues (52\%), the roles of team members (50\%) and safety and emergency procedures $(65 \%)$ and did not appear to be covered in the majority of the respondents' undergraduate. These results are summarised in Table 3.

Findings in respect of safety and emergency procedures are particularly disturbing if one considers that hospitalbased SLPs are frequently faced with complex, medically unstable dysphagia patients. Consequences such as choking, aspiration and malnutrition are therefore a daily reality for dysphagia clinicians in acute care settings (Mirro and Patey, 1991). It is thus essential for clinicians to have adequate knowledge of emergency and safety techniques in order to prevent or intervene in the event of medical complications (ASHA, 1987; CST, 1990). Although clinicians may often not be personally trained in emergency procedures, they still need to ensure that appropriate safety equipment and trained personnel are available during potentially dangerous situations (AASH, 1994).

Clinicians should also be aware of local policy and guidelines in the area of cross- infection (AASH, 1994; SASLHA, 1998). The limited number of respondents who reported receiving this input during their undergraduate training is very disconcerting. SASLHA (1998) lists routine precautions for infection control in a hospital setting but no specific attention is paid to the area of dysphagia. An added concern is the lack of attention in the document on the precaution against HIV transmission, especially in view of current health policy stressing that health care workers are in high risk situations for HIV transmission (College of Medicine of South Africa, 1991). It is therefore recommended that SASLHA use Kulpa's (1990) guidelines for SLPs with regard to safety precautions against HIV transmission and management of patients.

Practical clinical training at an undergraduate level was investigated according to the guidelines provided by CST (1990), ASHA (1991) and AASH (1994). According to Table 4 , the vast majority of the respondents reported that they did not receive any level of the recommended practical training at the undergraduate level. This is a disconcerting finding as these guidelines serve to ensure that students receive comprehensive clinical training. While it may be understandable that independent assessment and treatment of dysphagia patients may not be allowed due to the high risk nature of the area, this factor does not account for students not practicing techniques on normal subjects or even observing and assisting qualified professionals.

Based on these findings, it is not surprising that the overwhelming majority $(92 \%)$ of the respondents rated their undergraduate training as poor. The most common reason given by respondents was the lack of practical training included in their undergraduate courses. Similar findings were obtained in an ASHA Standards Validation Study which aimed to validate the professional domains, tasks, knowledge and skills in the general practice of speechlanguage pathology (cited in Bernthal and Bankson, 1992). Respondents recommended an increase in practical training hours in order to ensure that students have a variety of experience with individuals with various disorders of differing severity. Other reasons provided by respondents for rating their undergraduate training as poor, included the lack of a specific dysphagia module, the limited number of lectures on dysphagia, an insufficient amount of information covered theoretically, poor structuring of the course and a lack of exposure to teamwork.

\section{Postgraduate training}

Almost two-thirds of the respondents (64\%) in the study indicated that they had attended conferences, lectures or

TABLE 3. Theoretical areas in dysphagia covered at an undergraduate level $(N=48)$

\begin{tabular}{|l|c|c|c|}
\hline Areas & Covered in Courses & Not Covered in Courses & No Responses \\
\hline Anatomy / Physiology & $86 \%$ & $6 \%$ & $8 \%$ \\
\hline Medical Conditions & $73 \%$ & $21 \%$ & $6 \%$ \\
\hline Assessment & $79 \%$ & $13 \%$ & $8 \%$ \\
\hline Management & $75 \%$ & $17 \%$ & $8 \%$ \\
\hline Ethical Issues & $35 \%$ & $52 \%$ & $13 \%$ \\
\hline Safety Issues & $25 \%$ & $65 \%$ & $10 \%$ \\
\hline Roles of Team Members & $50 \%$ & 40 & $10 \%$ \\
\hline
\end{tabular}


workshops after graduation. Respondents' attendance at postgraduate courses indicates their awareness of their responsibility to continue their education after qualification in order to maintain a high standard of professional competence (SASLHA, 1997; SASLHA, 1998). Technological advances and ever- increasing knowledge bases demand that clinicians acquire current levels of expertise through various forms of continuing education.

Almost half $(47 \%)$ of the postgraduate courses attended by respondents were one day in length. More importantly, none of the courses extended for as long as a week and therefore did not comply with recommendations by Logemann (1995) in terms of length of course, namely 5-6 workshops, each of 2-3 days in length. Such courses are probably inappropriate in the South African situation where staff and financial resources for rehabilitation services are poor (White Paper for the Transformation of the Health System in South Africa, 1997). Clinicians therefore have to maximise existing resources by considering their access to the major providers of continuing professional education in speech-language pathology. Rassi and McElroy (1992a) identify these providers as being: a) college and universities; b) state and national professional associations and public agencies; c) hospitals and clinics, and d) independent providers such as publishers and equipment manufacturers.

The content included in the courses attended by the respondents was investigated according to the theoretical areas recommended by ASHA (1989), CST (1990) and AASH (1994). These results are summarised in Table 3.3. The vast majority of respondents $(94 \%)$ reported the inclusion of anatomy and physiology related to dysphagia. All the respondents (100\%) also reported that the procedures and techniques of dysphagia assessment and management were covered in their postgraduate courses. In contrast, only about half of the respondents reported the inclusion of interdisciplinary teaching and clinical practice on normal subjects and dysphagia patients. This finding is disconcerting as a lack of multidisciplinary management in dysphagia has been shown to lead to fragmented patient care (Lefton-Grief and Arvedson, 1997).

The limited inclusion of clinical practice with both normal subjects and with dysphagia patients in respondents' postgraduate courses is also of great concern in view of the restricted hands-on training received by most respondents during their undergraduate courses. This finding is not unique to the South African situation. Inherent in this finding is the lack of recognition that practical training plays a vital and integral role in the educational preparation of SLPs (Rassi and McElroy, 1992b). Respondents in the study thus apparently had limited opportunities for application of theoretical knowledge in the clinical setting during both undergraduate and postgraduate training. Once again, the

TABLE 4: Practical training in dysphagia at an undergraduate level $(\mathrm{N}=47)^{*}$

\begin{tabular}{|l|c|c|}
\hline \multicolumn{1}{|c|}{ PRACTICAL AREAS } & $\begin{array}{c}\text { COVERED IN } \\
\text { TRAINING }\end{array}$ & $\begin{array}{c}\text { NOT COVERED IN } \\
\text { TRAINING }\end{array}$ \\
\hline $\begin{array}{l}\text { Practising dysphagia assessment and } \\
\text { treatment with normal subjects }\end{array}$ & $4 \%$ & $96 \%$ \\
\hline $\begin{array}{l}\text { Observing dysphagia assessment and } \\
\text { treatment with dysphagia patients }\end{array}$ & $34 \%$ & $66 \%$ \\
\hline $\begin{array}{l}\text { Assisting with dysphagia assessment and } \\
\text { treatment of dysphagia patients under supervision }\end{array}$ & $15 \%$ & $85 \%$ \\
\hline $\begin{array}{l}\text { Independent dysphagia assessment and } \\
\text { treatment of dysphagia patients }\end{array}$ & $4 \%$ & $96 \%$ \\
\hline $\begin{array}{l}\text { Interdisciplinary dysphagia assessment and } \\
\text { treatment of dysphagia patients }\end{array}$ & $4 \%$ & $96 \%$ \\
\hline
\end{tabular}

* not applicable to 2 respondents; 1 respondent failed to respond

TABLE 5. Content of postgraduate courses in dysphagia $(\mathrm{N}=38)$

\begin{tabular}{|l|c|c|c|}
\hline AREAS & $\begin{array}{c}\text { COVERED } \\
\text { IN COURSES }\end{array}$ & $\begin{array}{c}\text { NOT COVERED } \\
\text { IN COURSES }\end{array}$ & $\begin{array}{c}\text { NO } \\
\text { RESPONSES }\end{array}$ \\
\hline Anatomy/physiology & $94 \%$ & $3 \%$ & $3 \%$ \\
\hline Assessment procedures \& techniques & $100 \%$ & $0 \%$ & $0 \%$ \\
\hline Management procedures and techniques & $100 \%$ & $0 \%$ & $0 \%$ \\
\hline Interdisciplinary teaching & $56 \%$ & $38 \%$ & $6 \%$ \\
\hline Clinical practice on normal subjects & $50 \%$ & $47 \%$ & $3 \%$ \\
\hline Clinical practice on dysphagia patients & $53 \%$ & $44 \%$ & $3 \%$ \\
\hline
\end{tabular}


findings highlight the need for the professional organisation for SLPs to develop a position paper on dysphagia so that appropriate standards of postgraduate courses can be established and maintained.

Only half of the respondents $(50 \%)$ who attended postgraduate training rated it as good. A further $44 \%$ of the respondents rated their training as average, while a limited number of respondents (6\%) felt that the training was poor. Various positive aspects of the postgraduate courses were described by respondents. The most common aspect related to the opportunity for practical training and the fact that the trainers were adequately qualified experts working in the field. Other positive factors included the adequacy of the course content in terms of material not covered at an undergraduate level, information on new advances in the field, input from various professionals and the relevance of material to the hospital environment. A focus on multidisciplinary work and counselling skills as well as specific training on interpreting normal and abnormal videofluroscopic studies was also described as being particularly beneficial. As with the undergraduate training, limitations of postgraduate courses described by respondents related most frequently to the lack of practical training included in the courses.

\section{ROLE OF THE SPEECH THERAPIST.}

With regard to the role of the SLP in dysphagia, the vast majority of the respondents showed an awareness that the SLP should be involved in all areas including identification of dysphagia patients (90\%), assessing and treating dysphagia patients (100\%), establishing and co-ordinating a multidisciplinary team (78\%), providing information to patients and caregivers $(100 \%)$,educating other health professionals about dysphagia (100\%) and conducting research on normal and abnormal swallowing (98\%). This finding was encouraging as these roles of the SLP are emphasized in the literature and by the various professional organizations.

The overwhelming majority of the respondents (90\%) felt that the role of the SLP in dysphagia management was only partially recognised by their medical colleagues. The most common reason cited by respondents for the lack of full recognition by medical colleagues related to the lack of knowledge not only of their role in dysphagia but also the general areas of work of a speech therapist. Respondents claimed that this lack of knowledge was evident in the limited number of referrals generally and the number of inappropriate referrals received. Respondents reported that referrals were made by a restricted number of doctors usually older doctors who had been educated by speech therapists over the years or newly trained doctors who had a rehabilitation week included in their undergraduate training.

Underlying reasons suggested by respondents for this poor knowledge included the lack of information on rehabilitation provided to doctors at an undergraduate level and the fact that most speech therapy departments were not situated at medical schools where doctors are trained. Respondents also felt that the limited numbers of speech therapists in the hospital setting as well as general time constraints severely restricted attendance at ward rounds and participation in teamwork as well as opportunities for initiating contact with doctors.

The majority of the respondents ( $71 \%$ ) felt that their role in dysphagia was only partially recognised by their paramedical colleagues. As with medical staff, the main reason offered by respondents for the lack of full recognition, related to the limited knowledge about the specific role of the speech therapist in dysphagia. Respondents felt that this lack of knowledge was reflected in the small number of paramedical staff who referred patients, the restricted number of referrals received, the fact that most dysphagia patients were initially referred only as speech/language patients, and the fact that only patients with severe dysphagia symptoms were referred.

These findings serve to highlight the importance of interdisciplinary teaching at an undergraduate level so that there is carryover into the work setting after qualification. Respondents also highlighted the critical need for clinicians to actively market their role in dysphagia practice.

\section{KNOWLEDGE AND PERCEPTIONS OF MULTIDISCIPLINARY TEAMWORK INDYSPHAGIA}

All the respondents $(100 \%)$ felt that teamwork was

TABLE 6: Role of the Speech-Language Pathologist in Dysphagia (N=50)

\begin{tabular}{|l|l|l|}
\hline ROLE OF THE SLP & YES & NO \\
\hline Identification of dysphagia patients & $90 \%$ & $10 \%$ \\
\hline Assessment of dysphagia patients & $100 \%$ & $0 \%$ \\
\hline Treatment of dysphagia patients & $100 \%$ & $0 \%$ \\
\hline Establishing a multidisciplinary team & $92 \%$ & $8 \%$ \\
\hline Co-ordinating a multidisciplinary team & $78 \%$ & $22 \%$ \\
\hline Providing information to patients and caregivers & $100 \%$ & $0 \%$ \\
\hline Education of other health professionals & $100 \%$ & $0 \%$ \\
\hline Conducting research on normal and abnormal swallowing & $98 \% *$ & $0 \%$ \\
\hline
\end{tabular}

*1 respondent did not respond 
necessary for assessing and managing dysphagia patients. Several investigators have in fact reported that comprehensive treatment by an interdisciplinary team has led to improved management of patients' nutritional status, reduced likelihood of complications, greater cost-effectiveness of treatment and earlier discharge of patients (Young and Durant-Jones, 1990).

Furthermore, the majority of respondents showed an awareness of which professionals comprise a multidisciplinary dysphagia team. The overwhelming majority of the respondents included all the professionals listed as part of the dysphagia team, namely dieticians, physiotherapists, occupational therapists, neurologists, doctors, nurses, radiologists and ear, nose and throat specialists.

\section{ACCESS TO ESSENTIAL RESOURCES}

Respondents' access to essential resources for dysphagia practice was investigated. These sources included various diagnostic procedures, suctioning equipment, diet modification services, dysphagia team members and supervision / mentor systems.

With regard to diagnostic procedures, the overwhelming majority of the respondents (98\%) indicated that they had access to $\mathrm{x}$-rays as a diagnostic procedure. Only approximately half of the respondents (54\%) indicated that they had access to videofluoroscopy. Almost one-third (32\%) did not have access, while a further $14 \%$ failed to respond to the question. This relatively high rate of a lack of response could be related to respondents being unaware of whether the diagnostic procedure was available or not, which could imply limited use of the procedure in dysphagia management. This finding is disconcerting as videofluoroscopy, specifically the modified Barium swallow has been described as the 'gold standard' in the evaluation and treatment of dysphagia (McHorney and Rosenbek, 1998). A number of implications arise from the responses furnished by clinicians in the study. Firstly, there is a need for respondents to be aware of the specific uses of videofluoroscopy in dysphagia assessment and treatment. Efforts also need to be made by respondents to find out about access to this diagnostic procedure. Lastly, if there is a lack of access to videofluoroscopy, respondents need to be aware of alternative diagnostic procedures as well as the need for more conservative managemen't as suggested by Groher (1995). Comprehensive knowledge of assessment procedures and techniques thus needs to be provided at an undergraduate level.

Many of the respondents (32\%) failed to respond to the question about access to ultrasound and a further $6 \%$ indicated that they were unsure of availability of this procedure in their work settings. Hence, it can be postulated from these results that limited use was made of this procedure as a diagnostic tool even if it was available. The results also suggest poor knowledge of instrumental procedures by many respondents. A very limited number of respondents $(38 \%)$ indicated that they had access to ultrasound. The unique characteristics of ultrasound make the procedure an important part of the comprehensive diagnosis of dysphagia. Limited access and possibly poor knowledge of the procedure thus places respondents and patients at a distinct disadvantage for more comprehensive holistic care. Increased understanding of instrumentation in dysphagia therefore needs to be facilitated at an undergraduate level.
Almost half of the respondents (46\%) failed to respond to the question about scintigraphy and a further $8 \%$ indicated that they were unsure of access to this procedure in their work settings. Once again, it can be postulated that this finding reflected limited knowledge of the procedure and limited use of it as a diagnostic tool even if it was available. Only $10 \%$ of the respondents indicated access to scintigraphy while $36 \%$ were sure about not having access to the procedure. This finding is not surprising particularly in the South African situation considering that scintigraphy is both costly and requires specialised nuclear medicine expertise (Sonies, 1991). The present focus of the South African Health Department is to shift expenditures and commission equipment for the delivery of primary health care services (White Paper for the Transformation of the Health System in South Africa, 1997). In line with this focus, national policy and guidelines have been developed for essential technology.

Similar findings in relation to previous diagnostic procedures were obtained with respect to Fibreoptic Endoscopic Examination of Swallowing (FEES). Almost half $(46 \%)$ of the respondents failed to respond while a further $10 \%$ indicated that they were unsure of access to FEES. An extremely limited number of respondents (8\%) indicated that they had access to FEES, while $36 \%$ did not have access to this procedure. The accuracy of this finding is questionable if one considers that this procedure is routinely used by ENTs for assessing the integrity of the laryngeal and pharyngeal structures (Logemann, 1998). It is therefore disconcerting that many of the respondents appeared to be unaware of access to this procedure and were therefore presumably making limited use of it as an effective tool for dysphagia management.

With regard to Electromyography (EMG), almost half of the respondents (46\%) failed to respond and a further $6 \%$ indicated that they were unsure of whether EMG was available in their work settings. A mere $10 \%$ of the respondents indicated access to EMG while more than onethird (38\%) were sure about inaccessibility. It is important for clinicians to be aware of the specific use of this diagnostic procedure as an assessment and therapeutic tool in dysphagia, especially in the presence of neuromuscular disorders. The procedure may also prove to be useful in the absence of other procedures, especially in the South African situation where resources are limited. There are therefore serious concerns regarding the large number of respondents who appeared to be unaware of the availability of EMG as well as the large number who did not have access to this resource. Appropriate referrals for specific patients are therefore compromised and this situation, in turn, may impact on early diagnosis and comprehensive care.

The results of access to manometry followed a very similar pattern to those for the other diagnostic procedures. The largest proportion of the respondents $(46 \%)$ did not respond to the question, while $8 \%$ indicated their uncertainty about access to manometry. Only $8 \%$ of the respondents indicated definite access to the procedure, while $38 \%$ clearly had no access to this resource.

Almost one-third of the respondents $(31 \%)$ indicated that they did not have access to suctioning equipment in their work settings. This result was surprising as one would have expected the standard availability of suction equipment in a hospital setting where it is regularly used by nurses and physiotherapists in patients with chest conditions. It can therefore be postulated that respondents had limited 
knowledge about suction equipment and were therefore unaware of available access to it. This finding is extremely worrying as suctioning can play a critical role during management of dysphagia patients. Limited knowledge of access to suction equipment suggests that respondents were not familiar with safety and emergency procedures in the event of choking or aspiration. This finding is not surprising in view of earlier findings that almost two-thirds (65\%) of the respondents reported that safety and emergency procedures were not covered in their undergraduate courses. Poor knowledge of these procedures has potentially lifethreatening implications for patients, especially those with chronic pulmonary disease. There is therefore an urgent need for undergraduate curricula in dysphagia to incorporate the area of safety and emergency procedures. Furthermore, a dysphagia position paper by SASLHA could serve to highlight potential risk factors in dysphagia and the necessary precautions that need to be undertaken.

The overwhelming majority of respondents (94\%) indicated that they had access to diet modification facilities. This finding is important for dysphagia management as diet modification is a specific strategy in the assessment and treatment of swallowing disorders. Standard hospital diets often pose problems for dysphagia patients.

The overwhelming majority of the respondents indicated that they had access to all the team members mentioned i.e. dietician, physiotherapist, occupational therapist, neurologist, doctor, nurse, radiologist and ENT.

The results indicate that more than half $(54 \%)$ of the respondents did not have any system of supervision/ mentoring operating in their departments. CST (1990) and AASH (1994) state that it is the medico-legal and ethical responsibility of SLPS who are aware that they lack the desired level of skill to seek guidance from more senior or experienced staff before intervening in management of the patient. The Principle of Ethics II (ASHA, 1994) clearly stipulates that clinicians should engage in only those aspects of the profession that are within the scope of their competence considering the level of their education, training and experience. SASLHA (1998) also states that negligence can be proved in instances where it is clear that intervention should have been undertaken by a more experienced clinician or the inexperienced therapist should have requested supervision. The lack of respondents' access to a system of supervision or mentoring in dysphagia management has serious implications. Without the institution of an organised system of supervision or mentoring, many of the respondents might not have been getting the necessary support and assistance from more experienced staff and the level of clinical service might not have been optimal for the patient. This possibility, in turn, raises questions about quality assurance at the work settings in the study.

Just under half of the respondents $(46 \%)$ indicated that there were systems of supervision or mentoring operating in their departments. There was a combination of both formal and informal systems being implemented at the time of the study, and this situation depended largely on the presence and level of experience of more senior staff. There appeared to be a specific focus in many departments on new graduates. These clinicians were either specifically assigned to senior supervisors or were simply made aware of available assistance whenever needed. Some of the respondents also reported that their heads of department and senior staff took responsibility for organising regular case discussions and continuing education and also encouraged new graduates to attend outside courses and conferences.

Rassi and McElroy (1992a) found that this responsibility of senior staff for more junior personnel was important as most professionals cannot easily identify their learning needs and therefore require guidance and assistance in structuring their continuing professional education.

An overwhelming majority (98\%) of the respondents felt that a system of supervision or mentoring for dysphagia treatment was important. They linked the need for supervision to the life-threatening nature of the work and many expressed concern about the safety of the patients. A number of the respondents also remarked on the challenging, stressful and frightening nature of dysphagia management. The lack of confidence and feeling of incompetence in new graduates and junior therapists was a frequently cited concern and these feelings were linked by many respondents to limited undergraduate training in dysphagia, especially with respect to the practical component. It was felt that supervision and mentoring would allow for the development of experience and confidence, as well as for accountability to the patient and the profession as a whole. Respondents felt that supervision would give therapists the opportunity to recognise their own limitations and thereby not compromise optimal care of the patient. The large caseload of dysphagia patients within hospitals was also of concern to respondents and the need for quality assurance measures was highlighted. In addition, respondents recognised that dysphagia management is a growing field with new advancements occurring all the time. Therefore the need for sharing information, knowledge and skills was vital. Respondents' concerns in this area highlight the need for a position paper in South Africa which could specify the need for supervision as formulated by CST (1990) and AASH (1994).

\section{KNOWLEDGE AND COMPETENCE}

\section{Knowledge}

Almost half of the respondent (46\%) rated their knowledge on human anatomy related to dysphagia as being average while a further $42 \%$ rated their knowledge as being good. This finding was not surprising as the majority of the respondents indicated that they had covered this aspect theoretically in their undergraduate training. In addition, the theory on anatomy was covered in $94 \%$ of the postgraduate courses attended by respondents.

The majority of the respondents $(58 \%)$ rated their knowledge of the risk factors in dysphagia as being good. This finding was not unexpected as the majority of the respondents $(70 \%)$ were exposed to the various medical conditions and disorders resulting in dysphagia at an undergraduate level.

The majority of the respondents rated their knowledge about instrumental procedures as poor (36\%) or average $(38 \%)$. Earlier results had indicated that assessment procedures and techniques had been covered in the majority of respondents' undergraduate and postgraduate courses. However, respondents' knowledge and awarenesś of access to specific instrumental procedures was found to be limited when access to resources was probed. This finding suggests that respondents' knowledge might have been restricted to commonly used procedures such as x-rays and videofluoroscopy and their limited knowledge about other procedures 
may have been highlighted by the question on access to resources.

The largest proportion of the respondents $(48 \%)$ rated their knowledge of alternate feeding methods as good. This finding could be anticipated in view of the fact that procedures and techniques of dysphagia management were reported to have been covered theoretically in the majority of the respondents' undergraduate training.

The majority of the respondents $(65 \%)$ rated their knowledge of the roles of key dysphagia team members as good. This finding can only be partially attributed to respondents' training as the roles of team members were reportedly covered in only approximately half of the respondents' undergraduate and postgraduate courses. It can be postulated that respondents' self-perceived 'good' knowledge of the roles was also related to their access and exposure to various team members in their work settings.

The majority of the respondents rated their knowledge about medical terminology and conditions associated with dysphagia as being either average (40\%) or good (42\%), i.e. only $18 \%$ rated their knowledge as poor. This finding is not surprising if one considers that this area was covered in the majority $(70 \%)$ of the respondents' undergraduate training.

\section{Skills}

Just under half of the respondents (47\%) rated their skills in assessing dysphagia patients as average i.e. a limited number of respondents $(29 \%)$ rated their skills as good. This finding was not surprising considering the fact that the vast majority of respondents reported limited opportunities for practical training in both undergraduate and postgraduate courses attended. These results suggest that the provision of theoretical knowledge without the accompanying practical experience was perceived by respondents as inadequate in terms of preparation of their skills for use in the workplace. This finding of a lack of practical training in dysphagia courses is well documented in the literature (Toner, 1995).

Inadequate skills with regard to assessing patients has serious implications for the safety of patients as clinicians need to make accurate diagnoses in order to implement appropriate treatment. The life-threatening risks of choking and aspirating demand a) high level of skill in assessment of dysphagia patients and highlights, once again, the need for position statements which guide effective training of students at an undergraduate level and clinicians at a postgraduate level.

As with the assessment of dysphagia patients, the largest proportion of the respondents $(49 \%)$ rated their skills in treating dysphagia patients as being average. Similar reasons can be postulated for this finding i.e. the limited practical hands-on training gained by the respondents during their undergraduate and postgraduate courses.

\section{Feelings of Emotional Comfort or Confidence}

The largest proportion of the respondents $(41 \%)$ rated their feelings of emotional comfort or confidence in handling $d y$ sphagia patients as average. Inadequate theoretical and practical training provided at courses attended, large case loads, limited knowledge of safety and emergency procedures and limited access to support systems from more experienced professionals were probably all factors contributing to decreased emotional comfort and confidence levels in handling dysphagia patients. A possible implication of such feelings is that clinicians may then not be willing to engage in active advocacy regarding the professionals' role in dysphagia and may also project a poor image of the profession by not being assertive in their knowledge, skills and decision-making. Probably one of the best ways of improving respondents' emotional comfort and confidence levels in handling patients is to ensure effective training at an undergraduate level.

The majority of the respondents rated their feelings of emotional comfort or confidence in counselling the families of dysphagia patients as being average (37\%) or good (39\%). A possible explanation for respondents being more comfortable or confident with counselling the families as compared to actual handling of patients is that respondents might have been experienced with counselling in general as it is incorporated regularly into all areas of work in speech and language pathology. Furthermore, respondents had generally rated their knowledge about dysphagia better than their skills in handling dysphagia patients. A large component of counselling families includes the sharing of information and therefore requires a good theoretical knowledge base.

\section{GENERAL VIEWS AND CONCERNS}

At the end of the questionnaire, respondents were given the opportunity to express their general views and concerns about the area of dysphagia. The types of concerns cited by respondents included inadequate training in dysphagia at an undergraduate level (particularly limited hands-on practical training and lack of knowledge of safety and emergency procedures); poor knowledge of the role of the SLP in dysphagia by other health professionals; limited resources available (including objective diagnostic instrumentation, suction equipment, postgraduate courses and continuing education, experienced contact people in the field, monthly support groups with more experienced clinicians, and guidance and supervision within the work setting); and the need for quality assurance measures.

Respondents' suggestions for overcoming these concerns related to improving undergraduate and postgraduate training, maximising limited resources and the need for policy documents.Considering dysphagia as only a postgraduate specialisation area was described by respondents as being impractical since all hospital speech therapists were faced with the ethical responsibility of treating dysphagia patients on a daily basis. In addition, it is recommended that supervision/mentoring systems be implemented using existing resources and expertise from both academic institutions and hospital departments.

More than half $(59 \%)$ of the respondents proposed strategies for dealing with the inadequacies of undergraduate training. It was felt that the course should be taught as a separate module which was structured to provide students with a firm theoretical grounding. Significantly more emphasis on the practical hands-on training in dysphagia assessment and treatment was suggested with both normal subjects and dysphagia patients. Respondents also felt that students should be involved in more clinical practicals at hospitals in order to gain experience in multidisciplinary dysphagia management. It was felt that students should also be taught about legal and ethical issues and be exposed to advances in instrumentation at an 
undergraduate level. In addition, respondents recommended a need for the university lecturers involved in teaching the area of dysphagia to be adequately experienced in the area, particularly on a practical level.

There was a strong consensus among respondents that all postgraduate courses include a practical component in addition to the theory. A system of observing speech therapists at other hospitals was also recommended. However, respondents did not distinguish between postgraduate training for degree or non-degree purposes.

In order to improve access to resources it was felt that support groups consisting of therapists from different hospitals would allow for case discussions and guidance. Developing a specialist list would also allow for easy contact with more experienced therapists whenever the need arose.

\section{CONCLUSION}

In conclusion, the results of this study suggest that many SLPs in South Africa may be involved in the area of dysphagia intervention. Findings have also indicated that there are possible gaps in clinicians' knowledge and skills in this area, which may be linked to inadequate training received at undergraduate and postgraduate levels, particularly with regard to practical hands-on training as well as theory on advances in instrumentation; ethical issues; safety and emergency procedures; and multidisciplinary management of dysphagia. Furthermore, clinicians are probably also faced with limited access to supervision systems as well as limited resources in general. These difficulties would appear to be further confounded by limited knowledge of their role in dysphagia by other health professionals and the subsequent need to actively market their services in their work settings. These problems faced by clinicians, in addition to the life-threatening nature of the work, highlights the need for clinicians, training institutions and the professional organisation for SLPs to make a concerted effort to improve the existing situation. However, possibly the most important reason to undertake such efforts is for the basic rights of dysphagia patients themselves. As health care workers,

"the concept of quality care is fundamental to the values, ethics and traditions of our profession - at the very least to do no harm; usually to do some good; and ideally to realise the greatest good possible in any given situation" [Frattali, 1990, p.39].

\section{Note}

The first author is a senior speech and hearing therapist employed at the Chris Hani Baragwanath Hospital and conducted this research project as a partial requirement for the degree M.A. Speech Pathology at the University of the Witwatersrand.

\section{References}

American Speech-Language-Hearing Association. (1985). Clinical Supervision in Speech-Language Pathology and Audiology. ASHA, 28 (6), 57-60.

American Speech-Language-Hearing Association. (1987). Ad Hoc Committee on Dysphagia report. ASHA, 29 (4), 57-58. American Speech-Language-Hearing Association. (1989).
Knowledge and Skills needed by Speech-Language Patho-logists Providing Services to Dysphagic Patients. ASHA, 32 (Suppl. 2); 7-12.

American Speech-Language-Hearing Association. (1991). Instrumental Diagnostic Procedures for Swallowing. ASHA, 33, 67-73

American Speech-Language-Hearing Association. (1993). Preferred Practice Patterns for the Professions of Speech-Language Pathology and Audiology. ASHA, 35 (Suppl.11), 1-100.

American Speech-Language-Hearing Association. (1994). Code of Ethics. ASHA, 36 (Suppl. 13), 1-2.

Australian Association of Speech and Hearing. (1994). Dysphagia Position Paper. Australia: AASH.

Bernthal, J.E. and Bankson, N.W. (1992). Higher Education. In J.A. Rassi and M.D. McElroy (Eds.) The Education of Audiologists and Speech-Language Pathologists. Maryland: York Press.

Cannito, M.P. (1995). Establishing a Dysphagia Curriculum at the University of Memphis: a personal perspective. ASHA Special Interest Divisions, 4 (2), 7-8.

College of Medicine of South Africa. (1991), Management of HIV positive patients. South African Medical Journal, 79, 688-690.

College of Speech Therapists. (1990). Position Paper on Dysphagia. U.K.: CS'T

Frattali, C.M. (1990). Quality Assurance Today: Learning the Basics. ASHA, 32, 39-40.

Groher, M.E. (1995). Preparing the Speech-Language Pathologist in Dysphagia Management: a serious problem with emerging solutions. ASHA Special Interest Divisions, 4 (2), 2-3.

Kulpa, J.I. (1990). AIDS / HIV: Implications for Speech-Language Pathologists and Audiologists. ASHA, 32, 46-48.

Langmore, S.E. (1991). Managing the Complications of Aspiration in Dysphagic Adults. Seminars in Speech and Language, 12 (3), 199-208.

Logemann, J. (1995). Education in Dysphagia. ASHA Special Interest Divisions, 4 (2), 3-5.

Logemann, J.A. (1998). Evaluation and Treatment of Swallowing Disorders. USA: Pro-Ed Publishers.

Martin, B.J.W. (1995). Dysphagia Evaluation and Management: Clinical Training, Clinical Competency and Speciality Recognition. The South African Journal of Communication Disorders, $42,3-6$.

McHorney, C.A. and Rosenbek, J.C. (1998). Functional Outcome Assessment of Adults with Oropharyngeal Dysphagia. Seminars in Speech and Language, 19 (3), 235-246.

Miller, R.M. (1995). APerspective on a Dysphagia Course: one man's view. ASHA Special Interest Divisions, 4 (2), 5-7.

Mirro, J.F. and Patey, C. (1991). Developing a Dysphagia Dietary Program. Seminars in Speech and Language, 12 (3), 218-227.

Preliminary Draft of Policy Document for Communication Professionals. (1993). S.A.: Bonaledi Nursing College.

Rassi, J.A. and McElroy, M.D. (1992a). Curriculum development. In J.A. Rassi and M.D. McElroy (Eds.) The Education of Audiologists and Speech-Language Pathologists. Maryland: York Press.

Rassi, J.A. and McElroy, M.D. (1992b). Education in the Clinic. In J.A. Rassi and M.D. McElroy (Eds.) The Education of Audiologists and Speech-Language Pathologists. Maryland: York Press.

Sonies, B.C. (1991). Instrumental Procedures for Dyspha'gia Diagnosis. Seminars in Speech and Language, 13 (3), 185-198.

South African Speech-Language Hearing Association. (1996). Newsletter. South Africa: SASLHA.

South African Speech-Language Hearing Association. (1997). Códe of Ethics. South Africa: SASLHA.

South African Speech-Language Hearing Association. (1998) Guidelines on Service Provision in Hospitals. South Africa: SASLHA.

Toner, M.A. (1995). Obstacles to Providing Dysphagia Training in a Small Academic Program. ASHA Special Interest Divisions, 4 (2), 8-9.

White Paper for the Transformation of the Health System in South Africa. 16 April 1997. Government Gazette Notice No. 667 of 1997. Pretoria: Government Printers.

Young, E.C. and Durant-Jones, L. (1990). Developing a Dysphagia Program in an Acute Care Hospital: A Needs Assessment. Dysphagia, 5, 159-165. 